\title{
Prevalence of Thyroid Nodules in China: A Health Examination Cohort-Based Study
}

OPEN ACCESS

Edited by:

Paolo Miccoli,

University of Pisa, Italy

Reviewed by:

Daniele Barbaro,

UO Endocrinologia ASL

Nord Ovest Toscana, Italy

Stefano Spiezia,

Local Health Authority

Naples 1 Center, Italy

*Correspondence:

Yi Ning

ningyi@vip.163.com

Guosheng Ren

rengs726@126.com

${ }^{t}$ These authors have contributed equally to this work

Specialty section: This article was submitted to

Thyroid Endocrinology,

a section of the journal

Frontiers in Endocrinology

Received: 04 March 2021

Accepted: 10 May 2021

Published: 26 May 2021

Citation:

Li Y, Jin C, Li J, Tong M, Wang M, Huang J, Ning Y and Ren G (2021)

Prevalence of Thyroid Nodules in China: A Health Examination Cohort-Based Study.

Front. Endocrinol. 12:676144. doi: 10.3389/fendo.2021.676144

\author{
Yunhai $\mathrm{Li}^{1 \dagger}$, Cheng $\mathrm{Jin}^{2,3+}$, Jie $\mathrm{Li}^{4 \dagger}$, Mingkun Tong ${ }^{2,3}$, Mengxue Wang ${ }^{4}$, Jiefeng Huang ${ }^{4}$, \\ Yi Ning ${ }^{2,3 *}$ and Guosheng Ren ${ }^{1,4 *}$ \\ ${ }^{1}$ Department of Endocrine and Breast Surgery, The First Affiliated Hospital of Chongqing Medical University, \\ Chongqing, China, ${ }^{2}$ Meinian Institute of Health, Beijing, China, ${ }^{3}$ Peking University Health Science Center, Meinian Public \\ Health Research Institute, Beijing, China, ${ }^{4}$ Chongqing Key Laboratory of Molecular Oncology and Epigenetics, The First \\ Affiliated Hospital of Chongqing Medical University, Chongqing, China
}

Background: Thyroid nodules are a common clinical problem and some are potentially cancerous; however, little is known about the prevalence of thyroid nodules in China. The objective of this study was to investigate the prevalence of thyroid nodules in a healthy Chinese population.

Methods: We reviewed electronic medical records of 13,178,313 participants from 30 provinces and regions who received health examinations and underwent thyroid ultrasound at Meinian Onehealth Healthcare in 2017. Among them, 6,192,357 were excluded based on predefined criteria. All thyroid nodules were diagnosed by ultrasonography, and standardized protocols were adopted for data collection, quality control, and data management.

Results: A total of 6,985,956 participants (mean age: $42.1 \pm 13.1$ years) were included in this study. The overall prevalence of thyroid nodules was 36.9\% (95\% Cl, 35.7\%-38.1\%; age- and sex-standardized prevalence 38.0\% [95\% Cl, 37.0\%-39.1\%]). The prevalence of thyroid nodules in females $(44.7 \%$ [95\% Cl, 43.4\%-45.9\%], age-standardized prevalence: $45.2 \%$ [95\% Cl, 44.1\%-46.4\%]) was significantly higher than that in males (29.9\% [95\% Cl, 28.8\%-31.0\%], age-standardized prevalence 31.2\% [95\% Cl, 30.1\%$32.2 \%$ ]; $P<0.001$ ). The prevalence of thyroid nodules decreased from $<18$ to 25 years, while increased with age over 25 years old. The top three provinces with the highest prevalence of thyroid nodules were Jilin (47.6\%), Liaoning (44.8\%), and Shandong (43.9\%), whereas Guizhou (23.9\%), Chongqing (26.2\%), and Shaanxi $(26.4 \%)$ had the lowest prevalence. Females had more than 10\% higher rates of thyroid nodules than males in all included provinces and regions, except for Tianjin (8.0\%). Based on the geographical regions of China, the northeast had the highest prevalence $(46.8 \%$ [95\% Cl, $44.1 \%-49.2 \%])$, whereas northwest had the lowest prevalence (28.9\% [95\% Cl, 26.9\%$31.6 \%]$. Based on multivariable logistic regression analysis, factors including age, gender, body mass index, systolic blood pressure, diastolic blood pressure, uric acid, fasting blood glucose, triglycerides, high-density lipoproteins, and low-density lipoproteins were significantly associated with the presence of thyroid nodules. 


\begin{abstract}
Conclusion: This study provides the first nationwide analysis of the prevalence of thyroid nodules in China. Our results showed that the prevalence of thyroid nodules was high in
\end{abstract} health screening Chinese people with regional-specific patterns.

Keywords: thyroid nodes, prevalence, risk factor, ultrasonography, China

\section{INTRODUCTION}

Thyroid nodules are defined as discrete lesions within the thyroid gland that are radiologically distinct from surrounding thyroid tissues (1). The diagnosis of thyroid nodules has become a common event in clinical practice worldwide. Currently, thyroid ultrasound plays a central role in the evaluation and management of thyroid nodules (2). With the increased use of high-resolution ultrasonography, thyroid nodules have been reported in $19 \%-67 \%$ of randomly selected individuals (3). Discovery of a thyroid nodule may be stressful for patients; however, more than $90 \%$ of thyroid nodules are benign lesions without symptoms (4). Moreover, a majority of nodules remain stable with regard to size during follow-up $(4,5)$.

Thyroid nodules are mainly derived from thyroid follicular cells; therefore, benign follicular nodules are the most common lesions. Follicular cell-derived thyroid cancers include papillary $(80 \%-85 \%)$, follicular $(10 \%-15 \%)$, poorly differentiated $(<2 \%)$, and undifferentiated (anaplastic; <2\%) (6). Thyroid nodule patients with some clinical factors, such as history of childhood irradiation, family history of thyroid cancer in a first-degree relative, male sex, and rapid nodule growth, are associated with increased risk of malignancy $(7,8)$. Although thyroid cancer screening may lead to earlier diagnosis, there is not adequate evidence to conclude that this would reduce morbidity or mortality $(9,10)$. Fine-needle aspiration cytology is the most sensitive and cost-effective method of identifying suspicious lesions that require surgery $(1,11)$. However, there is uncertainty concerning sonographically unsuspicious nodules and benign nodules indicated by fine-needle aspiration regarding the appropriate frequency of follow-up ultrasonography and the repetition of fine-needle aspiration. Overdiagnosis and overtreatment has become a challenge in the current management of thyroid nodules.

The prevalence of thyroid nodules in China has varied from $10.12 \%-46.56 \%$ among different studies (12). However, it is difficult to estimate the real prevalence of thyroid nodules, as China encompasses a big territorial area with diverse environments and a huge population composed of multiple races. In this study, we investigated the prevalence of thyroid nodules in China nationwide based on a health examination cohort with a large sample of $6,985,956$ individuals.

\section{METHODS}

\section{Study Cohort}

Records of all subjects who received health examinations at Meinian Onehealth Healthcare (MOH) in 2017 were reviewed.
$\mathrm{MOH}$ (Group) Co., Ltd was founded in 2004 and is a medical group providing preventive health screening services and doctor referrals in China. $\mathrm{MOH}$ has more than 600 professional medical centers in mainland China. Between January 1, 2017 and December 31, 2017, 13,178,313 participants received health examinations at $\mathrm{MOH}$ with accessible data. Approximately $46.7 \%$ ( $\mathrm{n}=6,151,025$ individuals) were excluded because they did not undergo thyroid ultrasound examination. Additionally, 41,119 patients with a prior history of malignancy were excluded to avoid influence from malignant disease. Subjects without age information $(n=213)$ were excluded because we suspected that the reliability of the data from these cases was low. Finally, a total of 6,985,956 individuals who received thyroid ultrasound examinations at least once were eligible for this analysis (Figure 1). Basic information including age, gender, and location were routinely collected for each participant based on a health examination reservation. The province of the individual was defined as the place where he or she received the health examination. This study was reviewed and approved by the Institutional Ethics Committees of the First Affiliated Hospital of Chongqing Medical University, in which written informed consent was waived. No participants received financial compensation.

\section{Clinical Examination and Laboratory Testing}

$\mathrm{MOH}$ used uniform criteria for clinical examinations and laboratory testing in all medical centers and guaranteed the uniformity of all health examination results. The thyroid of all included individuals was detected using a thyroid ultrasonography. All ultrasonographies were certified by China Food and Drug Administration (CFDA). Thyroid nodules were defined as discrete lesions in the thyroid gland that were radiologically distinct from the surrounding thyroid parenchyma, including solid, spongiform, cystic, and mixed nodules (1). Physical examination including height $(\mathrm{cm})$, weight $(\mathrm{kg})$, systolic blood pressure $(\mathrm{SBP},[\mathrm{mm} / \mathrm{Hg}])$, and diastolic blood pressure (DBP, $[\mathrm{mm} / \mathrm{Hg}])$ were measured twice, and averages were recorded. Body mass index (BMI) was calculated as weight $(\mathrm{kg})$ divided by height $(\mathrm{m})$ squared $\left(\mathrm{kg} / \mathrm{m}^{2}\right)$. Individuals were grouped into BMI $<18.5 \mathrm{~kg} / \mathrm{m}^{2}$ (underweight), $18.5-23.9 \mathrm{~kg} / \mathrm{m}^{2}$ (normal), $24.0-27.9$ $\mathrm{kg} / \mathrm{m}^{2}$ (overweight), and $\geq 28.0 \mathrm{~kg} / \mathrm{m}^{2}$ (obese) according to the guidelines for prevention and control of overweight and obesity in Chinese adults (13).

Blood samples were obtained by venipuncture after an 8 -h fast in the morning. The following factors were measured and available for analysis: triiodothyronine (T3), free triiodothyronine (FT3), thyroxine (T4), free thyroxine (FT4), thyroid-stimulating hormone (TSH), fasting blood glucose (FBG), hemoglobin Alc (HbAlc), uric acid (UA), triglycerides (TG), total cholesterol (TC), high-density lipoprotein (HDL), and low-density lipoprotein 


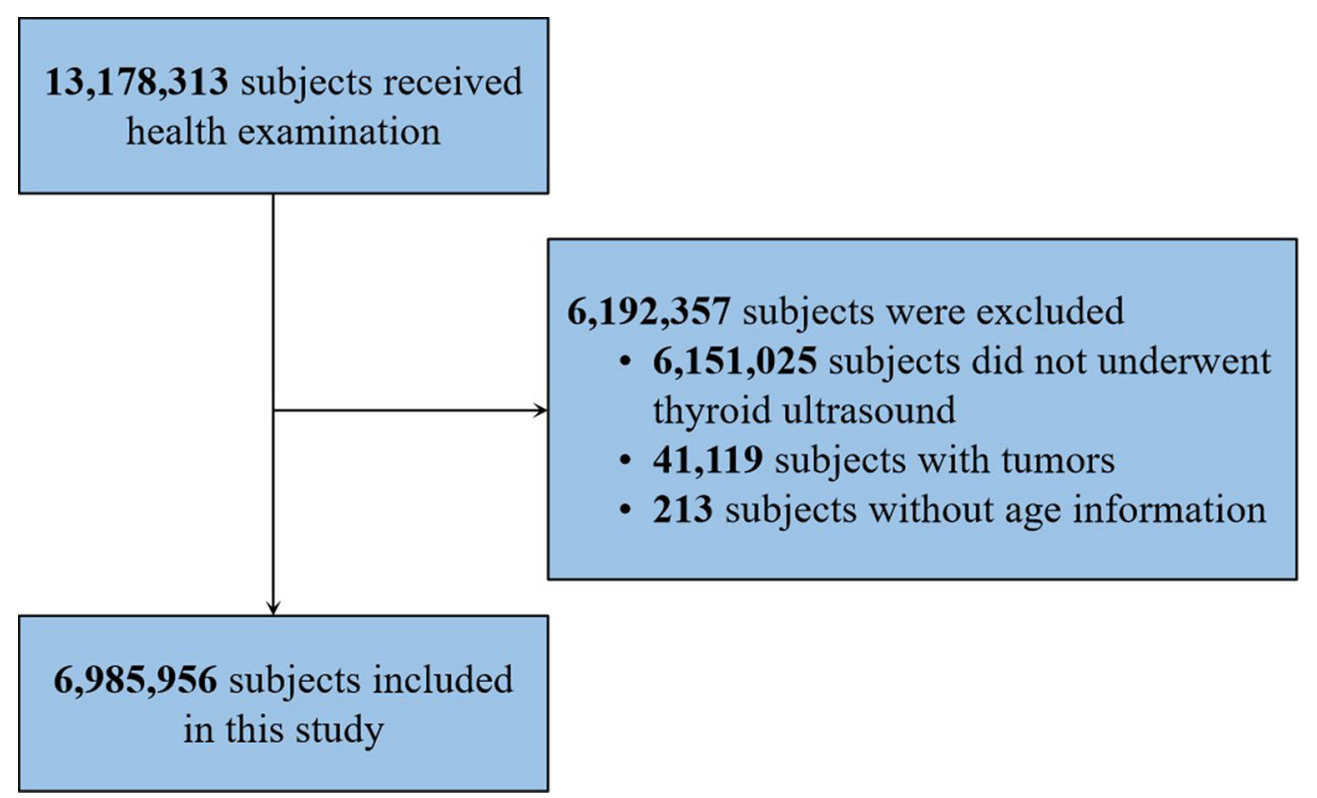

FIGURE 1 | Flowchart of subject selection.

(LDL). Participants were classified into high, normal, and low groups based on the normal ranges of these indicators.

\section{Statistical Analysis}

Demographic and baseline characteristics of all participants were analyzed using the chi-square test for categorical variables. The Mann-Whitney U test was used for continuous variables. Unknown data were not included in the analysis. The ageand/or sex-standardized prevalence of thyroid nodules was calculated using the data of the population distribution in China in 2010 by the direct method. Multivariable logistic regression analysis was used to explore potential risk factors associated with thyroid nodules. The model for multivariable logistic regression analysis included the following covariates: age, gender, BMI, SBP, DBP, UA, FBG, TG, HDL, and LDL. Height and weight were removed from the final model to avoid violating the principle of excluding linearly codependent variables because BMI was determined by them. To avoid missing data-related bias, T3, FT3, T4, FT4, TSH, and HbA1c were excluded because of the large amount of missing data. Odds ratios (ORs) and corresponding 95\% confidence intervals (CIs) were estimated based on the multivariable model. All tests were 2 -sided, and a $P$ value $<0.05$ was considered statistically significant. Statistical analyses were performed using SAS software, version 9.2 (SAS Institute Inc, Cary, NC).

\section{RESULTS}

\section{Study Cohort Characteristics}

A total of 6,985,956 individuals, who received thyroid ultrasonography while having a health examination at $\mathrm{MOH}$ in
2017, were included in this study (Table 1). The mean age of the participants was 42.1 years. Among the cases, 3,681,382 (52.7\%) were men and 3,304,574 (47.3\%) were women. All participants came from 30 provinces and regions of China including Anhui $(\mathrm{n}=180,624,2.6 \%)$, Beijing $(\mathrm{n}=191,834,2.7 \%)$, Chongqing $(\mathrm{n}=64,802,0.9 \%)$, Fujian $(\mathrm{n}=121,082,1.7 \%)$, Gansu $(\mathrm{n}=80,582$, $1.2 \%)$, Guangdong $(n=698,583,10.0 \%)$, Guangxi $(n=91,999$, $1.3 \%)$, Guizhou $(n=83,877,1.2 \%)$, Hainan $(n=40,282,0.6 \%)$, Hebei ( $n=228,498,3.3 \%)$, Henan $(n=542,813,7.8 \%)$, Heilongjiang $(\mathrm{n}=79,637,2.6 \%)$, Hubei $(\mathrm{n}=344,377,2.6 \%)$, Hunan $(\mathrm{n}=140,343$, $2.6 \%)$, Jilin ( $n=175,436,2.6 \%)$, Jiangsu $(n=360,573,2.6 \%)$, Jiangxi $(\mathrm{n}=84,497,1.2 \%)$, Liaoning $(\mathrm{n}=531,872,7.6 \%)$, Inner Mongolia $(n=130,261,1.9 \%)$, Ningxia $(n=21,837,0.3 \%)$, Qinghai $(n=10,461$, $0.1 \%)$, Shandong $(n=627,013,9.0 \%)$, Shanxi $(n=143,800,2.1 \%)$, Shaanxi ( $n=172,691,2.5 \%)$, Shanghai $(n=492,928,7.0 \%)$, Sichuan $(\mathrm{n}=553,015,7.9 \%)$, Tianjin $(\mathrm{n}=173,734,2.5 \%)$, Xinjiang $(n=107,938,1.5 \%)$, Yunnan $(n=225,327,3.2 \%)$, and Zhejiang $(n=285,240,4.1 \%)$. Thyroid nodules were identified in 2,578,156 participants. Participants with thyroid nodules were significantly older than those without nodules $(P<0.001)$. Thyroid nodules were more frequent in female participants than male $(P<0.001)$. Compared with participants without nodules, heights and weights were significantly lower in the cohort with nodules, while BMI, SBP, and DBP were higher in the group with nodules (Table 1). There were significant differences in the distributions of thyroid function tests (FT3, FT4, and TSH), glycemic parameters (FBG and HbA1c), UA, and serum lipids (TG, TC, HDL, and LDL) between individuals with and without thyroid nodules (Table 1).

\section{Prevalence of Thyroid Nodules}

The overall prevalence of thyroid nodules was $36.9 \%$ (95\% CI, $35.7 \%-38.1 \%)$. After standardizing by age and sex based on the 
TABLE 1 | Distribution of sociodemographic characteristics and blood factors among subjects with and without thyroid nodules.

\begin{tabular}{|c|c|c|c|c|c|c|}
\hline \multirow[t]{2}{*}{ Variables } & \multirow[t]{2}{*}{ Groups } & \multicolumn{2}{|c|}{ No nodules } & \multicolumn{2}{|c|}{ Nodules } & \multirow[t]{2}{*}{$P$-value } \\
\hline & & $N=4407938$ & (\%) & $N=2578231$ & $(\%)$ & \\
\hline Age & & $39.14 \pm 11.83$ & & $47.06 \pm 13.65$ & & $<0.001$ \\
\hline \multirow[t]{2}{*}{ Gender } & Male & 2579739 & $58.53 \%$ & 1101643 & $42.73 \%$ & $<0.001$ \\
\hline & Female & 1828061 & $41.47 \%$ & 1476513 & $57.27 \%$ & \\
\hline Height (cm) & & $165.95 \pm 8.53$ & & $163.80 \pm 8.57$ & & $<0.001$ \\
\hline Weight (kg) & & $66.08 \pm 13.17$ & & $65.87 \pm 12.50$ & & $<0.001$ \\
\hline BMI $\left(\mathrm{kg} / \mathrm{m}^{2}\right)$ & & $23.87 \pm 3.64$ & & $24.44 \pm 3.53$ & & $<0.001$ \\
\hline $\mathrm{SBP}(\mathrm{mm} / \mathrm{Hg})$ & & $121.46 \pm 17.29$ & & $126.23 \pm 19.78$ & & $<0.001$ \\
\hline DBP (mm/Hg) & & $74.80 \pm 11.98$ & & $76.58 \pm 12.41$ & & $<0.001$ \\
\hline \multirow[t]{4}{*}{ T3 } & Normal & 681457 & $98.79 \%$ & 525801 & $98.76 \%$ & $<0.001$ \\
\hline & Low & 4437 & $0.64 \%$ & 3696 & $0.69 \%$ & \\
\hline & High & 3920 & $0.57 \%$ & 2887 & $0.54 \%$ & \\
\hline & NA & 3717986 & & 2045772 & & \\
\hline \multirow[t]{4}{*}{ FT3 } & Normal & 412097 & $98.18 \%$ & 391844 & $98.37 \%$ & $<0.001$ \\
\hline & Low & 2079 & $0.50 \%$ & 1915 & $0.48 \%$ & \\
\hline & High & 5579 & $1.33 \%$ & 4566 & $1.15 \%$ & \\
\hline & NA & 3988045 & & 2179831 & & \\
\hline \multirow[t]{4}{*}{$\mathrm{T} 4$} & Normal & 682284 & $98.75 \%$ & 526902 & $98.77 \%$ & 0.64 \\
\hline & Low & 5144 & $0.74 \%$ & 3894 & $0.73 \%$ & \\
\hline & High & 3467 & $0.50 \%$ & 2681 & $0.50 \%$ & \\
\hline & NA & 3716905 & & 2044679 & & \\
\hline \multirow[t]{4}{*}{$\mathrm{FT} 4$} & Normal & 412111 & $97.21 \%$ & 392587 & $97.43 \%$ & $<0.001$ \\
\hline & Low & 4810 & $1.13 \%$ & 4594 & $1.14 \%$ & \\
\hline & High & 7029 & $1.66 \%$ & 5760 & $1.43 \%$ & \\
\hline & NA & 3983988 & & 2175290 & & \\
\hline \multirow[t]{4}{*}{$\mathrm{TSH}$} & Normal & 802817 & $92.89 \%$ & 622846 & $92.01 \%$ & $<0.001$ \\
\hline & Low & 11632 & $1.35 \%$ & 9624 & $1.42 \%$ & \\
\hline & High & 49815 & $5.76 \%$ & 44480 & $6.57 \%$ & \\
\hline & NA & 3543536 & & 1901206 & & \\
\hline \multirow[t]{4}{*}{ UA } & Normal & 3396466 & $80.60 \%$ & 2061211 & $83.72 \%$ & $<0.001$ \\
\hline & Low & 20094 & $0.48 \%$ & 13873 & $0.56 \%$ & \\
\hline & High & 797173 & $18.92 \%$ & 387035 & $15.72 \%$ & \\
\hline & NA & 194067 & & 116037 & & \\
\hline \multirow[t]{4}{*}{ FBG } & Normal & 3961576 & $92.79 \%$ & 2207015 & $88.10 \%$ & $<0.001$ \\
\hline & Low & 14580 & $0.34 \%$ & 6150 & $0.25 \%$ & \\
\hline & High & 293336 & $6.87 \%$ & 292080 & $11.66 \%$ & \\
\hline & NA & 138308 & & 72911 & & \\
\hline \multirow[t]{4}{*}{$\mathrm{HbA1c}$} & Normal & 414649 & $90.46 \%$ & 270613 & $84.84 \%$ & $<0.001$ \\
\hline & Low & 3457 & $0.75 \%$ & 1555 & 0.49\% & \\
\hline & High & 40262 & $8.78 \%$ & 46791 & $14.67 \%$ & \\
\hline & NA & 3949432 & & 2259197 & & \\
\hline \multirow[t]{4}{*}{$\mathrm{TG}$} & Normal & 3234098 & $74.78 \%$ & 1881437 & $74.08 \%$ & $<0.001$ \\
\hline & Low & 185335 & $4.29 \%$ & 94445 & $3.72 \%$ & \\
\hline & High & 905228 & $20.93 \%$ & 563856 & $22.20 \%$ & \\
\hline & NA & 83139 & & 38418 & & \\
\hline \multirow[t]{4}{*}{$\mathrm{TC}$} & Normal & 3494433 & $80.78 \%$ & 1947819 & $76.68 \%$ & $<0.001$ \\
\hline & Low & 44880 & $1.04 \%$ & 19618 & $0.77 \%$ & \\
\hline & High & 786602 & $18.18 \%$ & 572919 & $22.55 \%$ & \\
\hline & NA & 81885 & & 37800 & & \\
\hline \multirow[t]{4}{*}{ HDL } & Normal & 2885236 & $82.16 \%$ & 1760487 & $82.33 \%$ & $<0.001$ \\
\hline & Low & 168596 & $4.80 \%$ & 94576 & $4.42 \%$ & \\
\hline & High & 457958 & $13.04 \%$ & 283396 & $13.25 \%$ & \\
\hline & NA & 896010 & & 439697 & & \\
\hline LDL & Normal & 2919443 & $83.31 \%$ & 1736002 & $81.32 \%$ & $<0.001$ \\
\hline & Low & 107206 & $3.06 \%$ & 54228 & $2.54 \%$ & \\
\hline & High & 477757 & $13.63 \%$ & 344560 & $16.14 \%$ & \\
\hline & NA & 903394 & & 443366 & & \\
\hline
\end{tabular}

BMI, body mass index; SBP, systolic blood pressure; DBP, diastolic blood pressure; T3, triiodothyronine; FT3, free triiodothyronine; T4, thyroxine; FT4, free thyroxine; TSH, thyroidstimulating hormone; FBG, fasting blood glucose; HBA1C, hemoglobin A1C; UA, uric acid; TG, triglycerides; TC, total cholesterol; HDL, high-density lipoprotein; LDL, low-density lipoprotein; NA, Not available. 
population of China in 2010, the adjusted prevalence was $38.0 \%$ (95\% CI, 37.0\%-39.1\%). The crude nodule prevalence among female subjects was $44.7 \%$ (95\% CI, 43.4\%-45.9\%; agestandardized: $45.2 \%$ [95\% CI, 44.1\%-46.4\%]), which was significantly higher than males (29.9\% [95\% CI, 28.8\%-31.0\%]; age-standardized: 31.2\% [95\% CI, 30.1\%-32.2\%]); $P<0.001$ ). When analyzed by age, the prevalence of thyroid nodules was decreased in participants aged from $\leq 18(30.8 \%)$ to $26(20.5 \%)$, but increased over 26 years old in the overall cohort, up to $71.4 \%$ in subjects $\geq 80$ years old (Figure 2). In women, the prevalence was decreased from $\leq 18(35.9 \%)$ to $24(24.5 \%)$, and increased after 24 up to maximum $82.2 \%$ in female participants $\geq 80$ years. In men, the prevalence was lowest at 27 years old (16.2\%) and gradually increased to $65.9 \%$ in participants $\geq 80$ years. Among the 30 provinces and regions, the standardized prevalence of thyroid nodules ranged from $23.9 \%$ (Guizhou) to $47.6 \%$ (Jilin) in the overall cohort (Figure 3). Similar trends of the standardized prevalence were observed after stratifying individuals by gender (Figure 4). In most provinces and regions, women had 10\% higher prevalence of thyroid nodules than men, except for Tianjin, where women had an $8.0 \%$ higher prevalence rate. Ningxia had the largest difference of nodule prevalence between females and males (19.3\%), followed by Liaoning (18.2\%) and Anhui (17.0\%). The northeast of China had the highest nodule prevalence (46.8\% [95\% CI, 44.1\%-49.2\%]) followed by the north (41.2\% [95\% CI, 39.5\%-42.9\%]), east (38.6\% [95\% CI, 36.6\%-40.5\%]), central (37.4\% [95\% CI, $34.8 \%-40.0 \%])$, south $(30.5 \%$ [95\% CI, $28.3 \%-32.7 \%])$, southwest $(29.3 \%$ [95\% CI, 26.9\%-31.6\%]), and northwest (28.9\% [95\% CI, 26.9\%-31.6\%]). Coastal provinces had a significantly higher prevalence than inland provinces $(38.9 \%$ [95\% CI, 37.5\%-41.0\%] vs. $34.5 \%$ [95\% CI, 33.1\%-36.0\%]; $P<0.001)$.

\section{Baseline Variables Associated With Thyroid Nodules}

To determine factors related to thyroid nodules, we fit a multivariable logistic regression model, including age, gender, BMI, SBP, DBP, UA, FBG, TG, HDL, and LDL among the entire cohort (Table 2). Compared with individuals aged $\leq 18-25$, the

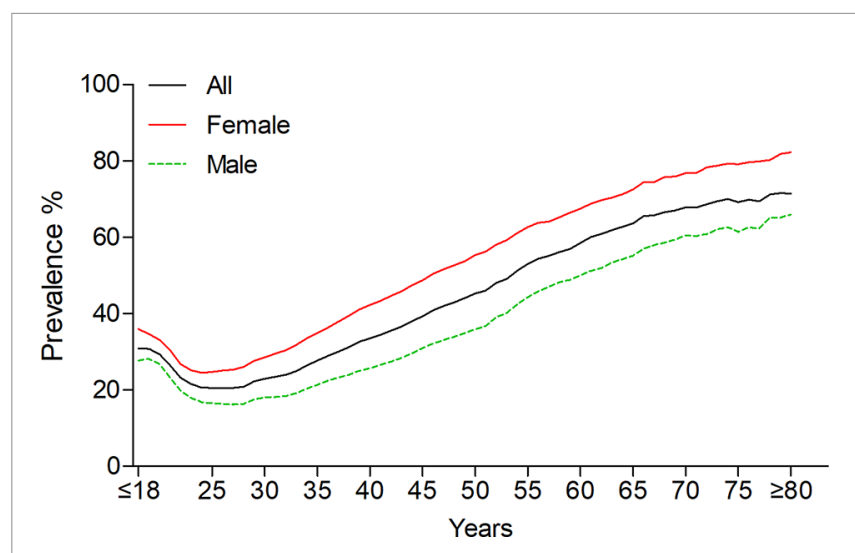

FIGURE 2 | Age-specific prevalence of thyroid nodules. odds of thyroid nodules were significantly increased with age $>25$ years. However, there was no obvious difference between the $\leq 18$ 25 and 26-40 groups in men (OR, 1.01; 95\% CI, 1.00-1.02; $P=$ 0.07 ). Women had more than 2 -fold higher odds (OR, 2.22; 95\% CI, 2.19-2.21; $P<0.001)$ compared with men. Individuals with BMI, SBP, DBP, and FBG values below the normal range were associated with significantly less odds of thyroid nodules, whereas those with higher BMI, SBP, DBP, and FBG were significantly associated with a greater risk of thyroid nodules. High UA levels were significantly associated with lower risk of thyroid nodules (OR, 0.98; 95\% CI, 0.98-0.99; $P<0.001$ ). Compared with participants who had normal levels of TG and HDL, TG presents a U-shape association with risk of thyroid nodules, whereas abnormal levels of HDL were significantly associated with decreased risk of thyroid nodules (Table 2). Low vs. normal LDL levels were significantly associated with decreased odds of thyroid nodules in the overall cohort (OR, 0.86; 95\% CI, 0.85$0.87 ; P<0.001$ ), as well as women (OR, 0.89; 95\% CI, 0.88-0.90; $P<0.001)$ and men (OR, 0.82; 95\% CI, 0.81-0.84; $P<0.001)$. High vs. normal LDL levels were associated with an increased risk of thyroid nodules in women (OR, 1.04; 95\% CI, 1.03-1.04; $P<$ $0.001)$; however, a decreased risk was observed in men with high LDL levels (OR, 0.98; 95\% CI, 0.97-0.98; $P<0.001$ ).

\section{DISCUSSION}

In this study, we systematically analyzed the prevalence of thyroid nodules among a huge cohort from 30 provinces and regions of China and revealed that the overall prevalence of thyroid nodules was $38.0 \%$. This is close to the prevalence in Korea (34.2\%), a neighboring country of China (14). However, we found that the prevalence of thyroid nodules varied from different provinces in China in a regional-specific manner. The northeast (Liaoning, Jilin, and Heilongjiang) and north (Beijing, Tianjin, Hebei, and Inner Mongolia) of China had a higher prevalence (over 40\%), whereas the southwest (Sichuan, Guizhou, Chongqing, and Yunnan) and northwest (Shaanxi, Ningxia, Gansu, Qinghai, and Xinjiang) of China had a lower prevalence (approximately 30\%). This prevalence pattern was different from a previous meta-analysis that pooled data between 2006 and 2013 in China (12). Additionally, the nodule prevalence of coastal provinces was higher than inland provinces. This regional-specific prevalence might result from multiple causes such as inheritance, environmental factors, and lifestyle.

Liaoning province had the second highest prevalence of thyroid nodules (44.8\%) in China, and our data suggested it was much higher than data reported in 2008 (approximately $10 \%)$ and $2009(38.5 \%)(15,16)$. The prevalence of thyroid nodules in Shanxi province was also higher in our study than in a previous study (17). Jiang et al. reported that the prevalence of thyroid nodules in Beijing was $40.1 \%$ based on 6,324 participants in 2016, which was close to our result (18). In Zhejiang province, the reported prevalence of thyroid nodules has varied from $22.5 \%$ to $38.4 \%(12,19)$, which was lower than the prevalence of $41.4 \%$ in this study $(n=285,240$ individuals from Zhejiang). However, these discrepancies cannot be 


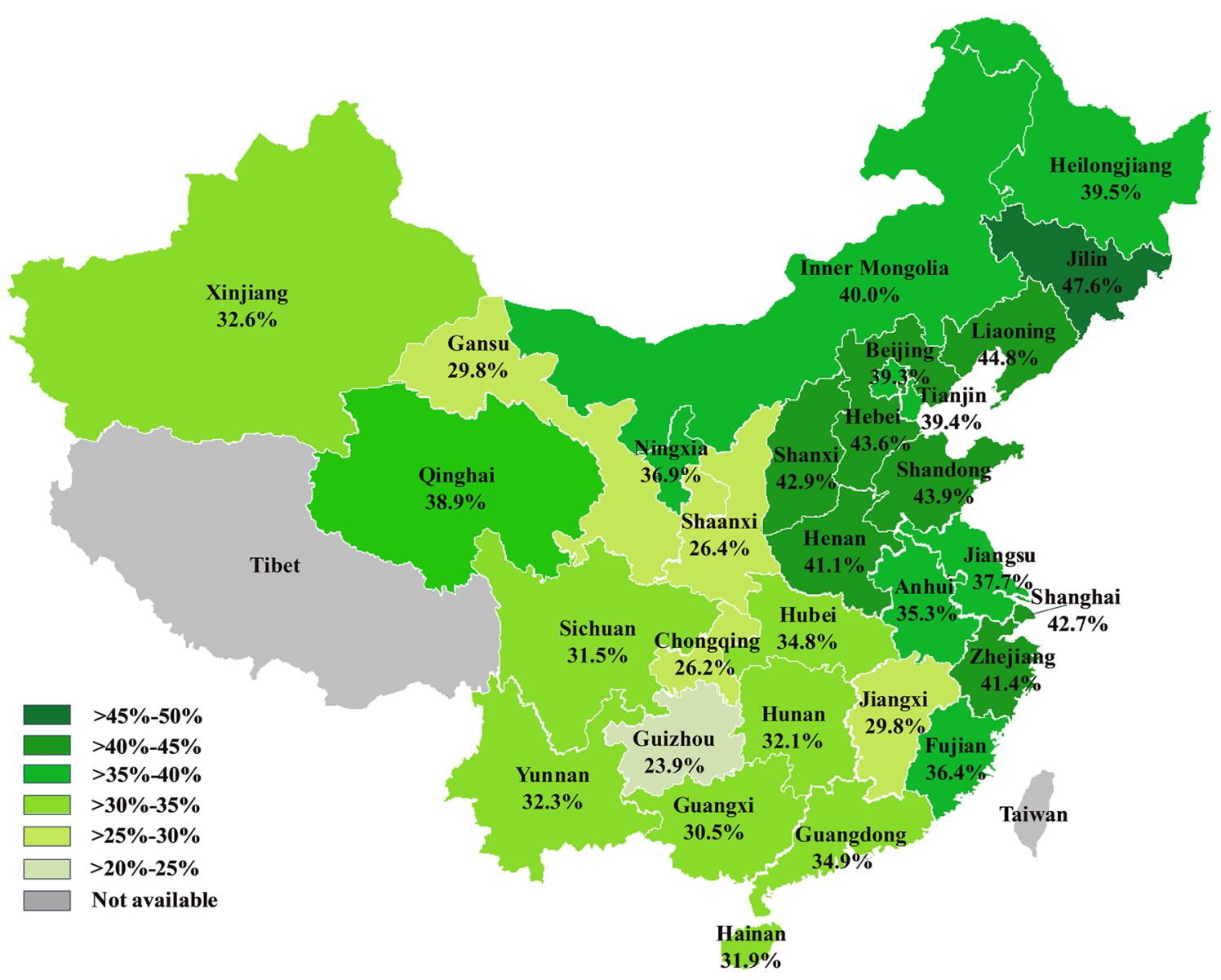

FIGURE 3 | The prevalence of thyroid nodules in 30 provinces and regions of China.

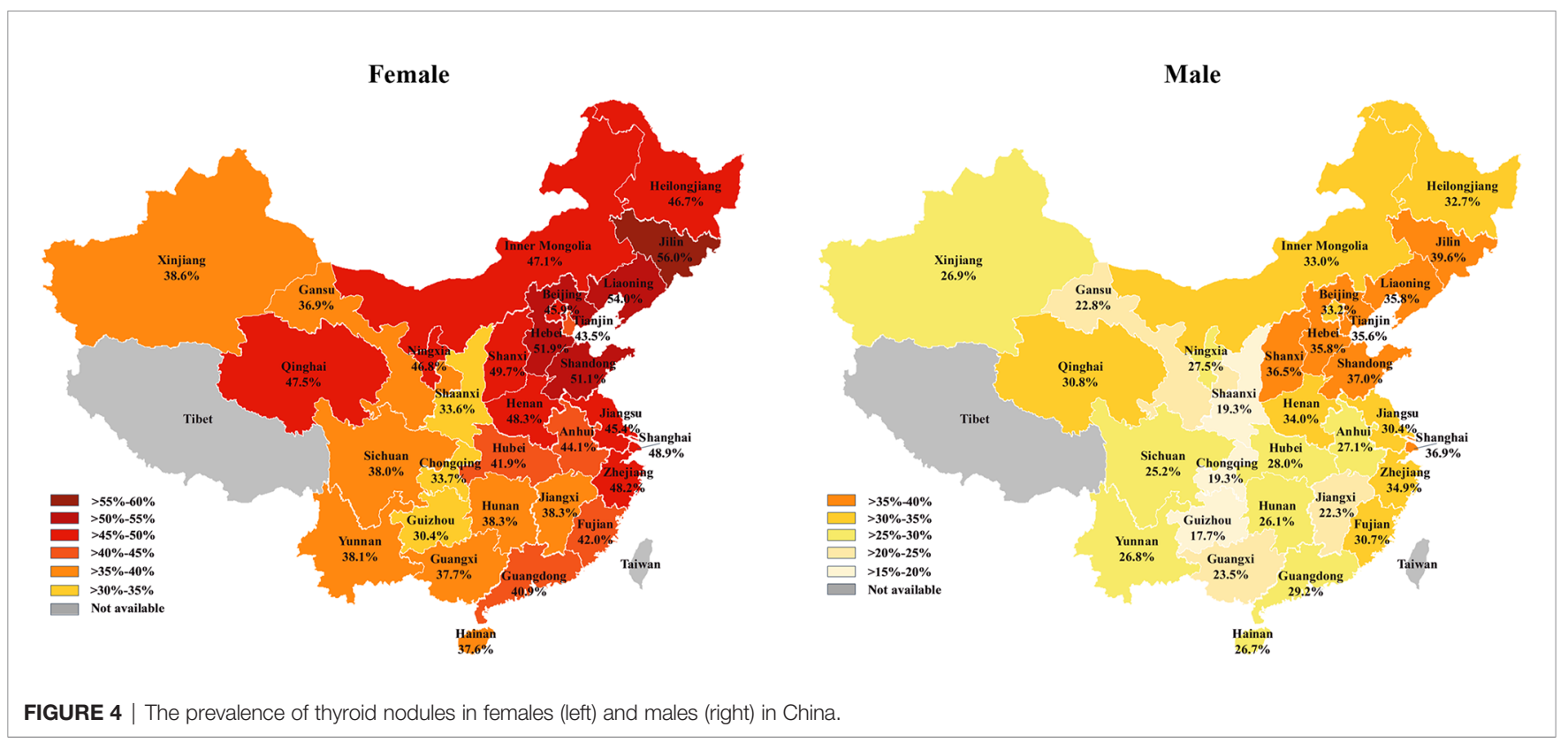


TABLE 2 | Multivariable logistic regression analysis.

\begin{tabular}{|c|c|c|c|c|c|c|c|}
\hline \multirow[t]{2}{*}{ Variables } & & \multicolumn{2}{|c|}{ All } & \multicolumn{2}{|c|}{ Female } & \multicolumn{2}{|c|}{ Male } \\
\hline & & OR (95\% Cl) & P-value & OR (95\% Cl) & P-value & OR (95\% Cl) & P-value \\
\hline \multirow[t]{5}{*}{ Age } & $\leq 18-25$ & reference & & reference & & reference & \\
\hline & $26-40$ & $1.16(1.15-1.17)$ & $<0.001$ & $1.29(1.27-1.30)$ & $<0.001$ & $1.01(1.00-1.02)$ & 0.07 \\
\hline & $41-55$ & $2.39(2.38-2.41)$ & $<0.001$ & $2.74(2.71-2.77)$ & $<0.001$ & $2.02(1.99-2.04)$ & $<0.001$ \\
\hline & $56-70$ & $4.61(4.59-4.67)$ & $<0.001$ & $5.02(4.96-5.08)$ & $<0.001$ & $4.08(4.03-4.12)$ & $<0.001$ \\
\hline & $\geq 71$ & 7.59 (7.49-7.69) & $<0.001$ & 8.48 (8.30-8.66) & $<0.001$ & $6.58(6.47-4.12)$ & $<0.001$ \\
\hline \multirow[t]{2}{*}{ Gender } & Male & reference & & NA & & NA & \\
\hline & Female & $2.20(2.19-2.21)$ & $<0.001$ & NA & & NA & \\
\hline \multirow[t]{5}{*}{ BMl } & $<18.5$ & $0.77(0.77-0.78)$ & $<0.001$ & $0.77(0.77-0.78)$ & $<0.001$ & $0.80(0.79-0.82)$ & $<0.001$ \\
\hline & $18.5-23.9$ & reference & & reference & & reference & \\
\hline & $24.0-27.9$ & $1.20(1.19-1.20)$ & $<0.001$ & $1.20(1.19-1.20)$ & $<0.001$ & $1.20(1.19-1.21)$ & $<0.001$ \\
\hline & $\geq 28.0$ & $1.32(1.32-1.33)$ & $<0.001$ & $1.30(1.29-1.31)$ & $<0.001$ & $1.34(1.33-1.35)$ & $<0.001$ \\
\hline & Unknown & $1.03(1.02-1.04)$ & $<0.001$ & $0.99(0.88-1.01)$ & 0.38 & 1.07 (1.06-1.08) & $<0.001$ \\
\hline \multirow[t]{4}{*}{ SBP } & $<90$ & $0.90(0.88-0.92)$ & $<0.001$ & $0.90(0.88-0.92)$ & $<0.001$ & $0.92(0.86-0.98)$ & 0.01 \\
\hline & $90-139$ & reference & & reference & & reference & \\
\hline & $\geq 140$ & $1.13(1.12-1.14)$ & $<0.001$ & $1.17(1.16-1.18)$ & $<0.001$ & $1.10(1.09-1.11)$ & $<0.001$ \\
\hline & Unknown & $1.16(0.96-1.40)$ & 0.13 & $1.14(0.92-1.42)$ & 0.23 & $1.25(0.85-1.84)$ & 0.25 \\
\hline \multirow[t]{4}{*}{ DBP } & $<60$ & $0.91(0.91-0.92)$ & $<0.001$ & $0.92(0.91-0.92)$ & $<0.001$ & $0.92(0.90-0.93)$ & $<0.001$ \\
\hline & $60-89$ & reference & & reference & & reference & \\
\hline & $\geq 90$ & 1.05 (1.05-1.06) & $<0.001$ & 1.07 (1.06-1.09) & $<0.001$ & $1.06(1.05-1.07)$ & $<0.001$ \\
\hline & Unknown & $0.93(0.77-1.13)$ & 0.48 & $0.96(0.77-1.19)$ & 0.71 & $0.86(0.59-1.27)$ & 0.45 \\
\hline \multirow[t]{4}{*}{ UA } & Normal & reference & & reference & & reference & \\
\hline & Low & $0.99(0.97-1.02)$ & 0.66 & 0.99 (0.99-1.02) & 0.40 & $1.00(0.97-1.04)$ & 0.83 \\
\hline & High & 0.98 (0.98-0.99) & $<0.001$ & $0.98(0.97-0.99)$ & $<0.001$ & 0.98 (0.98-0.99) & $<0.001$ \\
\hline & Unknown & $1.06(1.05-1.18)$ & $<0.001$ & $1.06(1.05-1.07)$ & $<0.001$ & $1.04(1.03-1.06)$ & $<0.001$ \\
\hline \multirow[t]{4}{*}{ FBG } & Normal & reference & & reference & & reference & \\
\hline & Low & $0.94(0.918-0.97)$ & $<0.001$ & $0.93(0.89-0.97)$ & $<0.001$ & $0.95(0.90-0.99)$ & 0.02 \\
\hline & High & $1.17(1.16-1.18)$ & $<0.001$ & $1.17(1.16-1.18)$ & $<0.001$ & $1.18(1.17-1.19)$ & $<0.001$ \\
\hline & Unknown & $0.99(0.98-1.00)$ & .42 & $1.00(0.99-1.02)$ & 0.77 & $0.97(0.96-0.99)$ & $<0.001$ \\
\hline \multirow[t]{4}{*}{ TG } & Normal & reference & & reference & & reference & \\
\hline & Low & $1.05(1.04-1.06)$ & $<0.001$ & $1.03(1.02-1.04)$ & $<0.001$ & 1.07 (1.06-1.09) & $<0.001$ \\
\hline & High & $1.02(1.01-1.02)$ & $<0.001$ & 1.04 (1.03-1.05) & $<0.001$ & $1.01(1.01-1.02)$ & $<0.001$ \\
\hline & Unknown & $0.90(0.89-0.91)$ & $<0.001$ & $0.91(0.89-0.93)$ & $<0.001$ & $0.89(0.87-0.91)$ & $<0.001$ \\
\hline \multirow[t]{4}{*}{ HDL } & Normal & reference & & reference & & reference & \\
\hline & Low & $0.98(0.97-0.98)$ & $<0.001$ & $0.94(0.92-0.95)$ & $<0.001$ & $0.99(0.98-1.00)$ & 0.05 \\
\hline & High & $0.90(0.90-0.91)$ & $<0.001$ & $0.94(0.93-0.95)$ & $<0.001$ & $0.85(0.84-0.85)$ & $<0.001$ \\
\hline & Unknown & $1.02(0.98-1.06)$ & 0.31 & $1.02(0.96-1.07)$ & 0.57 & $1.02(0.96-1.07)$ & 0.55 \\
\hline \multirow[t]{4}{*}{ LDL } & Normal & reference & & reference & & reference & \\
\hline & Low & $0.86(0.85-0.87)$ & $<0.001$ & $0.89(0.88-0.90)$ & $<0.001$ & $0.82(0.81-0.84)$ & $<0.001$ \\
\hline & High & $1.00(1.00-1.01)$ & 0.23 & $1.04(1.03-1.04)$ & $<0.001$ & $0.98(0.97-0.98)$ & $<0.001$ \\
\hline & Unknown & $0.91(0.88-0.95)$ & $<0.001$ & $0.93(0.88-0.98)$ & 0.004 & $0.91(0.86-0.96)$ & $<0.001$ \\
\hline
\end{tabular}

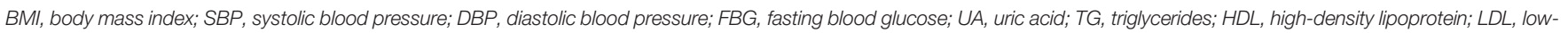
density lipoprotein; NA, Not available.

interpreted as changes in the inheritance and environmental factors, but be dramatic changes of lifestyle over past decade. In addition, the different sampling methods might also have impacted these results. Nevertheless, due to the reliability of our study population and the considerable number of participants, we believe that the present study is more reliable in reflecting the actual prevalence of thyroid nodules in China.

Gender and age are two critical factors associated with the prevalence of thyroid nodules (20). Consistent with previous studies, the frequency of thyroid nodules was significantly higher in female than that in male participants $(14,18,21)$. However, it was unexpected that the age-specific prevalence of thyroid nodules was decreased with age from $\leq 18$ to 26 years and increased in those older than 26 years, a phenomenon that was distinct from other studies, which concluded that the frequency of thyroid nodules increases with age $(14,18,20-22)$. These reports might have missed this phenomenon because of limitations of sample size and grouping individuals by decade, whereas our result was supported by an investigation that included a relatively large cohort (17). In the study by Liu et al., they found that the frequency of nodules showed a Ushaped curve with age increase and the group aged 20-30 years had the lowest prevalence (17). Together, these results indicated that the events of thyroid nodule disappearance exceed new nodules development during the age $\leq 18-26$ years. In contrast, a recent study that included 992 thyroid nodule patients with mean age 52.4 years and 5-year follow-up, showed that new thyroid nodules occurred in $93(9.3 \%)$ patients, whereas only one nodule disappeared during follow-up (5). However, further studies focused on young individuals are warranted to reveal the natural history of thyroid nodules and to clarify the underlying mechanisms with respect to thyroid nodules that spontaneously shrink or disappear. 
The multivariable logistic regression analysis indicated that age and gender were two independent risk factors associated with the development of thyroid nodules, which was consistent with other studies (21). Women had a 2.2-fold increased risk of developing thyroid nodules than men. Moreover, previous studies have reported that pregnancy was associated with new thyroid nodule formation and increased nodule size $(23,24)$. These results suggested that the development of thyroid nodules may be influenced by sex-related hormones such as estrogen and progesterone. Higher BMI was associated with an increased risk of thyroid nodules, whereas participants with a BMI lower than normal had a decreased risk of nodules. High FBG levels were an independent risk factor for thyroid nodules. It was consistent with the finding that thyroid nodule development is associated with obesity, insulin resistance, prediabetes, and diabetes (2528). In addition, central obesity, defined by a gender-specific waist circumference cut-off, has been demonstrated to be another independent risk factor for thyroid nodules (29). In this study, we found that blood pressure, UA, and serum lipids (TG, HDL, and LDL) were significantly associated with nodule disease. Although other studies have also shown similar relationships of thyroid nodules with hypertension, hyperuricemia, and serum lipids, the underlying mechanisms through which hypertension and serum lipids promote the development of thyroid nodules remain unclear $(27,30,31)$.

Iodine intake has been suspected to be a major dietary factor affecting the development of thyroid nodules, but the evidence remains inconclusive. In 1995, to prevent iodine deficiency disorders, the Chinese government launched a Universal Salt Iodization program, which directly resulted in a dramatic decrease of goiters. However, the number of cases with thyroid nodules, thyroid cancer, and hyperthyroidism increased over the same period. Therefore, some experts hold the point of view that iodine intake might be excessive in the Chinese population, especially in coastal provinces. Nevertheless, the reality is that individuals with deficient, adequate, and excessive iodine intake have prevalence of thyroid nodules $23.7 \%$, 53.8\% and $18.0 \%$, respectively, as reported by a previous study (32). Participants with high or excessive iodine intake did not show an increased risk of thyroid nodules, whereas deficient iodine intake was significantly associated with an increased risk of nodules (32). Similarly, participants consuming iodized salt had a lower prevalence of thyroid nodules than those consuming noniodized salt (21). However, they found that the prevalence of thyroid nodules increased when the urinary iodine concentrations were $>500 \mu \mathrm{g} / \mathrm{L}$. Regrettably, data regarding iodized salt intake and urinary iodine concentrations were not available for this study. Although we found a significant difference in the nodule prevalence between coastal and inland provinces, this cannot be simply explained by eating seafood, which is rich in iodine. Therefore, further investigations into the relationship between iodine and thyroid nodules are needed in the future.

This study had several limitations. First, the participants who received health examinations at $\mathrm{MOH}$ cannot represent the overall Chinese population due available data from real world health screening practice. In addition, $46.7 \%$ participants without thyroid ultrasound examination were excluded. These may result in sample selection bias. Second, the size, number and ultrasound imaging characteristics of thyroid nodules were not recorded in all medical records when we extracted thyroid nodules using a computerized keyword extraction method, we could not analyze nodule size, nodule number, and ultrasound imaging characteristics, such as microcalcification and sonogram echoes. Third, there were no biopsy data for sonographically suspicious nodules or follow-up for all individual included in this study. Therefore, the proportion of thyroid cancer among these nodules and the natural history of the thyroid nodules were unclear. Despite these limitations, to our knowledge, this study represents the first investigation of the prevalence of thyroid nodules nationwide in China using a huge sample size. We believe that these results appropriately reflect the disease status of thyroid nodules in China.

In summary, the results of this study suggested that the prevalence of thyroid nodules was high in the Chinese population and showed a regional-specific pattern. Thyroid nodules were also more prevalent among women than men. The prevalence of thyroid nodules was decreased from $\leq 18$ to 26 years and increased with age $>26$ years in the overall cohort. Factors including age, gender, BMI, SBP, DBP, UA, FBG, TG, HDL, and LDL were independently associated with development of thyroid nodules. These findings enhance our understanding of thyroid nodules in China.

\section{DATA AVAILABILITY STATEMENT}

The raw data supporting the conclusions of this article will be made available by the authors, without undue reservation.

\section{ETHICS STATEMENT}

The studies involving human participants were reviewed and approved by Institutional Ethics Committees of the First Affiliated Hospital of Chongqing Medical University. Written informed consent from the participants' legal guardian/next of kin was not required to participate in this study in accordance with the national legislation and the institutional requirements.

\section{AUTHOR CONTRIBUTIONS}

GR and YL designed the study. GR and YN supervised the study. YN, CJ, and MT had full access to all of the data in the study. CJ and MT conducted the statistical analysis. YL, JL, and MW drafted the manuscript. JH and YN revised the manuscript. All authors contributed to the article and approved the submitted version. 


\section{REFERENCES}

1. Haugen BR, Alexander EK, Bible KC, Doherty GM, Mandel SJ, Nikiforov YE, et al. 2015 American Thyroid Association Management Guidelines for Adult Patients With Thyroid Nodules and Differentiated Thyroid Cancer: The American Thyroid Association Guidelines Task Force on Thyroid Nodules and Differentiated Thyroid Cancer. Thyroid (2016) 26(1):1-133. doi: 10.1089/ thy.2015.0020

2. Valderrabano P, McGettigan MJ, Lam CA, Khazai L, Thompson ZJ, Chung $\mathrm{CH}$, et al. Thyroid Nodules With Indeterminate Cytology: Utility of the American Thyroid Association Sonographic Patterns for Cancer Risk Stratification. Thyroid (2018) 28(8):1004-12. doi: 10.1089/thy.2018.0085

3. Tan GH, Gharib H. Thyroid Incidentalomas: Management Approaches to Nonpalpable Nodules Discovered Incidentally on Thyroid Imaging. Ann Intern Med (1997) 126(3):226-31. doi: 10.7326/0003-4819-126-3-199702010-00009

4. Durante C, Grani G, Lamartina L, Filetti S, Mandel SJ, Cooper DS. The Diagnosis and Management of Thyroid Nodules: A Review. Jama (2018) 319 (9):914-24. doi: 10.1001/jama.2018.0898

5. Durante C, Costante G, Lucisano G, Bruno R, Meringolo D, Paciaroni A, et al. The Natural History of Benign Thyroid Nodules. Jama (2015) 313(9):926-35. doi: 10.1001/jama.2015.0956

6. Dralle H, Machens A, Basa J, Fatourechi V, Franceschi S, Hay ID, et al. Follicular Cell-Derived Thyroid Cancer. Nat Rev Dis Primers (2015) 1:15077. doi: 10.1038/nrdp.2015.77

7. Burman KD, Wartofsky L. Clinical Practice. Thyroid Nodules. N Engl J Med (2015) 373(24):2347-56. doi: 10.1056/NEJMcp1415786

8. Wong R, Farrell SG, Grossmann M. Thyroid Nodules: Diagnosis and Management. Med J Aust (2018) 209(2):92-8. doi: 10.5694/mja17.01204

9. Bibbins-Domingo K, Grossman DC, Curry SJ, Barry MJ, Davidson KW, Doubeni CA, et al. Screening for Thyroid Cancer: US Preventive Services Task Force Recommendation Statement. Jama (2017) 317(18):1882-7. doi: 10.1001/ jama.2017.4011

10. Sosa JA, Duh QY, Doherty G. Striving for Clarity About the Best Approach to Thyroid Cancer Screening and Treatment: Is the Pendulum Swinging Too Far? JAMA Surg (2017) 152(8):721-2. doi: 10.1001/jamasurg.2017.1338

11. Danese D, Sciacchitano S, Farsetti A, Andreoli M, Pontecorvi A. Diagnostic Accuracy of Conventional Versus Sonography-Guided Fine-Needle Aspiration Biopsy of Thyroid Nodules. Thyroid (1998) 8(1):15-21. doi: 10.1089/ thy.1998.8.15

12. Zhao W, Han C, Shi X, Xiong C, Sun J, Shan Z, et al. Prevalence of Goiter and Thyroid Nodules Before and After Implementation of the Universal Salt Iodization Program in Mainland China From 1985 to 2014: A Systematic Review and Meta-Analysis. PloS One (2014) 9(10):e109549. doi: 10.1371/ journal.pone.0109549

13. Chen C, Lu FC. The Guidelines for Prevention and Control of Overweight and Obesity in Chinese Adults. BioMed Environ Sci (2004) 17 Suppl:1-36. doi: 10.1111/j.1365-2028.2008.00991.x

14. Moon JH, Hyun MK, Lee JY, Shim JI, Kim TH, Choi HS, et al. Prevalence of Thyroid Nodules and Their Associated Clinical Parameters: A Large-Scale, Multicenter-Based Health Checkup Study. Korean J Intern Med (2018) 33 (4):753-62. doi: 10.3904/kjim.2015.273

15. Pan XF, Sun XY, Jia XD, Xu F, Zhao T, Jiang T, et al. Thyroid Nodular Diseases in the Population Indergoing Medical Examination and the Analysis of its Relative Factors in Dalian City,Liaoning Province. Chin J Endemiol (2009) 28(5):568-71. doi: 10.3760/cma.j.issn.1000-4955.2009.05.030

16. Yu X, Fan C, Shan Z, Teng X, Guan H, Li Y, et al. A Five-Year Follow-Up Study of Goiter and Thyroid Nodules in Three Regions With Different Iodine Intakes in China. J Endocrinol Invest (2008) 31(3):243-50. doi: 10.1007/bf03345597

17. Liu Y, Lin Z, Sheng C, Zhu Y, Huang Y, Zhong N, et al. The Prevalence of Thyroid Nodules in Northwest China and its Correlation With Metabolic Parameters and Uric Acid. Oncotarget (2017) 8(25):41555-62. doi: 10.18632/ oncotarget. 14720

18. Jiang H, Tian Y, Yan W, Kong Y, Wang H, Wang A, et al. The Prevalence of Thyroid Nodules and an Analysis of Related Lifestyle Factors in Beijing
Communities. Int J Environ Res Public Health (2016) 13(4):442. doi: 10.3390/ ijerph 13040442

19. Xu W, Chen Z, Li N, Liu H, Huo L, Huang Y, et al. Relationship of Anthropometric Measurements to Thyroid Nodules in a Chinese Population. BMJ Open (2015) 5 (12):e008452. doi: 10.1136/bmjopen-2015-008452

20. Mazzaferri EL. Management of a Solitary Thyroid Nodule. $N$ Engl J Med (1993) 328(8):553-9. doi: 10.1056/nejm199302253280807

21. Fan L, Tan L, Chen Y, Du C, Zhu M, Wang K, et al. Investigation on the Factors That Influence the Prevalence of Thyroid Nodules in Adults in Tianjin, China. J Trace Elem Med Biol (2018) 50:537-42. doi: 10.1016/ j.jtemb.2018.03.004

22. Kwong N, Medici M, Angell TE, Liu X, Marqusee E, Cibas ES, et al. The Influence of Patient Age on Thyroid Nodule Formation, Multinodularity, and Thyroid Cancer Risk. J Clin Endocrinol Metab (2015) 100(12):4434-40. doi: 10.1210/jc.2015-3100

23. Kung AW, Chau MT, Lao TT, Tam SC, Low LC. The Effect of Pregnancy on Thyroid Nodule Formation. J Clin Endocrinol Metab (2002) 87(3):1010-4. doi: 10.1210/jcem.87.3.8285

24. Alexander EK, Pearce EN, Brent GA, Brown RS, Chen H, Dosiou C, et al. Guidelines of the American Thyroid Association for the Diagnosis and Management of Thyroid Disease During Pregnancy and the Postpartum. Thyroid (2017) 27(3):315-89. doi: 10.1089/thy.2016.0457

25. Anil C, Akkurt A, Ayturk S, Kut A, Gursoy A. Impaired Glucose Metabolism is a Risk Factor for Increased Thyroid Volume and Nodule Prevalence in a Mild-to-Moderate Iodine Deficient Area. Metabolism (2013) 62(7):970-5. doi: 10.1016/j.metabol.2013.01.009

26. Sousa PA, Vaisman M, Carneiro JR, Guimaraes L, Freitas H, Pinheiro MF, et al. Prevalence of Goiter and Thyroid Nodular Disease in Patients With Class III Obesity. Arq Bras Endocrinol Metabol (2013) 57(2):120-5. doi: 10.1590/S0004-27302013000200004

27. Feng S, Zhang Z, Xu S, Mao X, Feng Y, Zhu Y, et al. The Prevalence of Thyroid Nodules and Their Association With Metabolic Syndrome Risk Factors in a Moderate Iodine Intake Area. Metab Syndr Relat Disord (2017) 15(2):93-7. doi: 10.1089/met.2016.0077

28. Buscemi S, Massenti FM, Vasto S, Galvano F, Buscemi C, Corleo D, et al. Association of Obesity and Diabetes With Thyroid Nodules. Endocrine (2018) 60(2):339-47. doi: 10.1007/s12020-017-1394-2

29. Song B, Zuo Z, Tan J, Guo J, Teng W, Lu Y, et al. Association of Thyroid Nodules With Adiposity: A Community-Based Cross-Sectional Study in China. BMC Endocr Disord (2018) 18(1):3. doi: 10.1186/s12902-0180232-8

30. Shin J, Kim MH, Yoon KH, Kang MI, Cha BY, Lim DJ. Relationship Between Metabolic Syndrome and Thyroid Nodules in Healthy Koreans. Korean J Intern Med (2016) 31(1):98-105. doi: 10.3904/kjim.2016.31.1.98

31. Ding X, Xu Y, Wang Y, Li X, Lu C, Su J, et al. Gender Disparity in the Relationship Between Prevalence of Thyroid Nodules and Metabolic Syndrome Components: The SHDC-CDPC Community-Based Study. Mediators Inflamm (2017) 2017:8481049-. doi: 10.1155/2017/8481049

32. Chen Z, Xu W, Huang Y, Jin X, Deng J, Zhu S, et al. Associations of Noniodized Salt and Thyroid Nodule Among the Chinese Population: A Large Cross-Sectional Study. Am J Clin Nutr (2013) 98(3):684-92. doi: $10.3945 /$ ajcn.112.054353

Conflict of Interest: The authors declare that the research was conducted in the absence of any commercial or financial relationships that could be construed as a potential conflict of interest.

Copyright $\odot 2021$ Li, Jin, Li, Tong, Wang, Huang, Ning and Ren. This is an openaccess article distributed under the terms of the Creative Commons Attribution License (CC BY). The use, distribution or reproduction in other forums is permitted, provided the original author(s) and the copyright owner(s) are credited and that the original publication in this journal is cited, in accordance with accepted academic practice. No use, distribution or reproduction is permitted which does not comply with these terms. 